\title{
Circular dichroism studies of low molecular weight hydrogelators: the use of SRCD and addressing practical issues.
}

\author{
Efstratios D. Sitsanidis, ${ }^{[a]}$ Carmen C. Piras, ${ }^{[a]}$ Bruce D. Alexander, ${ }^{[b]}$ Giuliano Siligardi, ${ }^{[c]}$ Tamas \\ Javorfi, ${ }^{[c]}$ Andrew J. Hall, ${ }^{[a]}$ and Alison A. Edwards ${ }^{*[a]}$
}

\begin{abstract}
Circular dichroism (CD) spectroscopy has been used extensively for the investigation of the conformation and configuration of chiral molecules, but its use for evaluating the mode of self-assembly in soft materials has been limited. Herein, we report a protocol for the study of such materials by electronic circular dichroism (CD) spectroscopy using commercial/benchtop instruments and synchrotron radiation (SR) using the B23 beamline available at Diamond Light Source. The use of B23 beamtime for SRCD was advantageous due to the unique enhanced spatial resolution achieved because of its highly collimated and small beamlight cross-section (ca $250 \mu \mathrm{m}$ ) and higher photon flux in the far UV region (175-250 nm) enhancing the signal-to-noise ratio relative to benchtop CD instruments. A set of low molecular weight (LMW) hydrogelators, comprising two Fmoc-protected
\end{abstract}

enantiomeric monosaccharides and one Fmoc-dipeptide (Fmoc-FF) were studied. The research focused on the optimization of sample preparation and handling, which then enabled the characterisation of sample conformational homogeneity and thermal stability. CD spectroscopy, in combination with other spectroscopic techniques and microscopy, will allow a better insight into the self-assembly of chiral building blocks into higher order structural architectures.

Keywords: Supramolecular hydrogels, self-assembly, circular dichroism, spectral profile.

\section{INTRODUCTION}

Low molecular weight (LMW) hydrogels are semi-solid viscoelastic materials composed of at least $99 \%$ water. Recently, the development of such materials has gained significant prominence due to their desirable physical properties and tuneable characteristics, ${ }^{1}$ leading to potential applications in areas such as catalysis, ${ }^{2,3}$ nanofabrication, ${ }^{4}$ sensing, ${ }^{5}$ tissue engineering, ${ }^{6-8}$ cell culture, ${ }^{9-11}$ wound healing ${ }^{12,13}$ and drug delivery. ${ }^{14-16}$ These soft materials arise from the self-assembly of small molecules in water via a number of intermolecular noncovalent interactions, such as hydrogen bonding, $\pi-\pi$ stacking, $\mathrm{CH}-\mathrm{m}$ bonds, ${ }^{17,18}$ metal coordination, van der Waals forces and solvophobic effects. It is these interactions that are responsible for the self-assembly which results in the gelation process and the encapsulation of solvent molecules within a three-dimensional fibrous network. ${ }^{19}$ There are several reports that describe the diverse nature of hydrogelators that can give rise to LMW hydrogels, where many have chiral components in their structure. ${ }^{20-26}$ The most common sources of intrinsic chirality are from amino acids and carbohydrates.

It is the molecular packing of the building blocks (self-assembly) to create a gel that gives rise to a circular dichroism (CD) spectrum which is characteristic for the gel (Figure 1). This is due to the configurational alignment of the formed fibres, leading to higher order architectures (matrix). For example, the gelator in solution often has a slightly higher absorbance (since it is a true solution) compared to its corresponding gel. This is because the gelator typically forms a very weak solution/suspension due to its poorer solubility in the solvent that it gels. By contrast, the $C D$ spectrum of the solution is typically much weaker (or negligible) compared to that of the gel where the signals arise from the interaction of the chromophores in their self-assembled state. In solution, where the molecules are moving freely, the overall exciton coupling between the chromophores is significantly lower. This is in marked contrast to the organisation of the higher order architecture of the gel. Therefore, CD is commonly used to observe the process of gel formation by supramolecular assembly, where researchers tend to speak about the solution (or sol) to gel process, i.e. sol-gel or gelation process. Since LMW hydrogels assemble via non-covalent interactions, the sol-gel process can be readily reversed, e.g. by thermal destruction to create the corresponding solution/suspension (i.e. gel-sol process).

[a] Mr ED Sitsanidis, Dr CC Piras, Dr AJ Hall, Dr AA Edwards Medway School of Pharmacy

Universities of Greenwich and Kent at Medway

Anson Building, Central Avenue, Chatham Maritime, Kent,

ME4 4TB, UK

Fax: (+)44 1634883927

E-mail: A.A.Edwards@kent.ac.uk

[b] Dr BD Alexander

Department of Pharmaceutical, Chemical \& Environmental Sciences

University of Greenwich,

Central Avenue, Chatham, Kent, ME4 4TB, UK

[c] Dr G Siligardi, Dr T Javorfi

Diamond Light Source

Didcot, Oxfordshire, OX11 ODE, UK

Received: ((will be filled in by the editorial staff))

Revised: ((will be filled in by the editorial staff) Published online: ((will be filled in by the editorial staff)) 


\section{INSERT FIGURE ONE HERE}

FIGURE 1 The utilisation of CD for the study of LMW gels.

Examination of literature to establish appropriate protocols for the study of LMW hydrogels by CD revealed a limited number of protocols and a wide variation of methods. ${ }^{27-30}$ We undertook a "bottom up" approach to measure the CD spectra of LMW hydrogels. In this study, previously reported structurally related Fmoc hydrogelators 1, 2 and 3 (Figure 2) were used for the development of a robust protocol to investigate molecular alignment in soft materials ${ }^{17,31,32}$ based on CD spectroscopy. For measurements of samples in solution, CD spectroscopy characterises the anisotropy and the intrinsic chirality of the chiral sample, whereas for hydrogels there is also the contribution of the anisotropy of the achiral media that is generated by circular birefringence (CB), linear dichroism (LD) and linear birefringence (LB). The CD measured with commercial instruments, due to the large beamlight cross section, can measure only the average of the chirality of the sample. To assess whether LD, LB and CB contributions were negligible or dominant, synchrotron radiation circular dichroism (SRCD) measurements can be conducted for several small spots, $0.5 \mathrm{~mm}$ in diameter, along the hydrogel specimens. The use of the vertical sample chamber of the $\mathrm{B} 23$ beamline ${ }^{33}$ made it possible to measure a hydrogel laid horizontally, without the complication of thickness change due to gravity (Figure 3). ${ }^{34}$

\section{INSERT FIGURE TWO HERE}

FIGURE 2 Structurally related hydrogelators GalNHFmoc 1, GlcNHFmoc 2 and Fmoc-FF 3.

\section{INSERT FIGURE THREE HERE}

FIGURE 3 Sample positioning in the sample chamber of module A, with a rotating platform and cylindrical cell holder, facilitated the acquisition of spectral data at three different sites of the hydrogel sample to allow evaluation of sample homogeneity as "spots".

Extensive optimization of both the sample preparation and handling enabled the development of a series of experiments to: (i) identify the presence of optical artefacts (LD and LB); (ii) observe the homogeneity of the samples; and (iii) monitor both the thermal disruption and reformation of the hydrogels (sol-gel reversibility). Due to time constraints of beamtime for this study, spectra were only acquired at rotations of $0^{\circ}$ and $90^{\circ}$ therefore only LD and LB were evaluated for these samples. CB ran be readily evaluated by comparison of spectra at $0^{\circ}$ and $180^{\circ}$ rotation of a given sample. This paper should provide a practical method for both experienced CD users and soft materials researchers for the study of chiral hydrogel materials.

\section{MATERIALS AND METHODS}

\section{Sample preparation}

Compounds $\mathbf{1}$ and $\mathbf{2}$ were synthesized as reported previously. ${ }^{17}$ Compound 3 was purchased from Biogelx (Newhouse, UK) and used as supplied. For the preparation of hydrogels, the following solvents were used: purified water (Romil, Super Purity) and phosphate buffered saline (PBS) solution which was prepared by dissolving PBS tablets (Sigma Aldrich, UK) in purified water. Solutions of the gelator were prepared using methanol (Romil, Super Purity).

\section{Solution samples}

Solution samples of hydrogelators 1-3 were obtained using methanol to allow a true solution to be obtained and suitable UV transparency as shown in Figure 7 (and Figures A and B of SI).

\section{Gel samples}

The gelation process and handling of the prepared hydrogels was optimised prior to any CD measurement, as was the method to obtain the hydrogel within the cuvette cell (see section 2 of SI). To avoid CD signal saturation, quartz cylindrical non-demountable cells (Hellma, style 121) with a range of path lengths were used $(0.5,0.2$ or $0.1 \mathrm{~mm})$. This was achieved by judicious choice of path length to give an optimal absorbance intensity (about 0.9 ). For the reduction of absorbance intensity, dilution of the hydrogels was not possible, as this would disrupt their self-assembly, therefore for each sample the minimum gelation concentration was kept constant.

For the sample preparation, two gelation methods were employed, namely sonication triggered gelation and thermally triggered gelation. It is noted that the gelation process used herein differs to that previously reported. ${ }^{17}$ All samples were gelled within cylindrical cells at a similar time (i.e. typically 24 hours before data acquisition) and in situ (e.g. at the Diamond Light Source premises) to minimize any manipulation that could affect the selfassembly. Hydrogels of $\mathbf{1}$ and 2 (A-D) were prepared, as described in Table 1, under conditions known to pass the gravitational tube inversion test. The four conditions employed were also utilised for Fmoc-FF 3 but only gelation by sonication in PBS was successful, thus providing hydrogel $\mathbf{E}$.

$2.0 \mathrm{mg}$ of the hydrogelator $(\mathbf{1}, \mathbf{2}$ or 3$)$ was weighed into a vial and $1.0 \mathrm{~mL}$ of water or PBS solution was added. The resulting suspension was then sonicated and transferred to a cell with a pipette.

Method for sonication triggered gelation

The cells were then left undisturbed overnight before the acquisition of SRCD and CD spectra.

Method for thermally triggered gelation (heating and cooling cycles)

The cells were placed in a block heater at $55^{\circ} \mathrm{C}$. The temperature was raised by $10{ }^{\circ} \mathrm{C}$ at ten-minute intervals until $95^{\circ} \mathrm{C}$ was obtained. The cooling process was performed in a controlled manner by reducing the temperature by $20^{\circ} \mathrm{C}$ every hour until room temperature was reached. Cells were then left undisturbed (in the block heater) overnight before acquisition of SRCD and CD spectra.

Method for thermal disruption of hydrogels $\boldsymbol{A}, \boldsymbol{B}$ and $\boldsymbol{E}$ and their reformation

After the CD spectrum of the hydrogel was obtained, the sample was heated at $85^{\circ} \mathrm{C}$ for approximately 30 minutes using a Peltier temperature controlled cell holder to reverse the self-assembly (i.e. destroy the gel) and a corresponding CD spectrum obtained at room temperature. The sample was then left at room temperature overnight (to allow gelation to re-occur) and the CD spectrum obtained to evaluate the reversibility of gelation, i.e. the gel-solgel cycle.

\section{INSERT TABLE ONE HERE}

TABLE 1 Gelation conditions. The concentration of all samples was $2.0 \mathrm{mg} / \mathrm{mL}$ ( ${ }^{*} T$ he hydrogelator also gels under these conditions but was not included in this study).

\section{Data acquisition}

CD spectra were firstly obtained using a Chirascan spectrophotometer for the evaluation of the absorbance intensity of the samples and gelation success, i.e. by visual inspection of the cell and the CD intensity. Module A at Diamond Light Source was used to acquire the SRCD spectra. A rotating platform combined with a cylindrical cell holder was used in module $A$ (Figure 3 ) to allow sampling of a selected area of the hydrogel in different orientations (i.e. $0^{\circ}$ and $90^{\circ}$ ) for the determination of the presence of optical artefacts, such as linear dichroism and linear birefringence. This sampling arrangement also enabled spectra to 
be recorded at three different hydrogel sites ("spots") of the same sample to allow evaluation of the homogeneity of the hydrogel (Figure 3). Where time allowed, the hydrogel sample was then finally heated at $85{ }^{\circ} \mathrm{C}$ for approximately 30 minutes using a Peltier temperature-controlled cell holder to reverse the selfassembly (i.e. break the gel) and allow a corresponding CD spectrum to be obtained. This facilitated interpretation of the CD and SRCD spectra of the corresponding hydrogel. Comparison of the CD and SRCD spectra obtained for the same hydrogel sample also facilitated the evaluation of any spectral differences due to the differing cross-sectional area of the light. A baseline spectrum was recorded for every hydrogel and solution sample in each instrument by the recording a spectrum of the solvent in the same cuvette cell, i.e. water or PBS for hydrogels and methanol for solutions as appropriate. All spectra were recorded at room temperature.

\section{Processing of the acquired spectra}

The absorbance spectrum of the sample in the Chirascan (and thus CD spectrum) was truncated where the absorbance exceeded 1.0 AU, thus necessitating the use of short path lengths. For the SRCD spectra of each sample, the absorbance spectrum was obtained of spot 2 for each sample and used to judge the spectral cut-off. Practical aspects meant that absorbance was only recorded on spot 2 (of the three spots). Therefore, on module A the PMT (HV) spectrum (for a given SRCD spectrum) was used to judge spectral cut-off ( $>600 \mathrm{~V}$ ) for each spot (see Figure $\mathrm{C}$ of SI).

Subtraction of water or PBS baseline spectra from the corresponding hydrogel spectra gave the corresponding baseline corrected spectra that are presented herein. For the methanolic solutions of the gelator, the corresponding methanol baseline spectrum was subtracted.

\section{RESULTS AND DISCUSSION}

The initial approach of the SRCD study targeted the optimization of sample preparation and data acquisition procedures to allow $C D$ to support the characterization of the self-assembly of the synthesized hydrogelators $\mathbf{1}$ and $\mathbf{2}$. After much optimisation, it was found that the best procedure was to form the hydrogels within the cells and that non-demountable cylindrical cells were most advantageous due to the range of path lengths available (see section 2 of $\mathrm{SI}$ ). In this manner, the hydrogel was not perturbed upon transfer. The hydrogels formed from $\mathbf{1}$ and $\mathbf{2}$ in both PBS solution and in water are summarised in Table 1. The judicious use of both $C D$ and SRCD experiments enabled the characterization of their gelation behaviour.

Similar experiments were also undertaken on the dipeptide hydrogelator 3 (Fmoc-FF). Although studied by CD, ${ }^{32,35}$ to the best of our knowledge, no SRCD information has been reported for hydrogels of $\mathbf{3}$. Comparison between the Fmoc-sugar hydrogels (1 and 2) and the Fmoc-dipeptide (3) is of interest since $\mathbf{3}$ has ionic character (from a $-\mathrm{COOH}$ moiety) and is known to hydrogel by a combination of $\pi-\pi$ and hydrogen bonding interactions. The sugar-containing hydrogels $\mathbf{1}$ and $\mathbf{2}$ have no ionic character and self-assemble by $\mathrm{CH}-\pi$ interactions. ${ }^{17}$ Any difference in the spectral features or behaviour may be characteristic of (i) the structural difference after due consideration of the contribution of the additional aromatic and carbonyl chromophores of the dipeptide motif and/or (ii) the differing self-assembly mechanisms. Based on the obtained CD information for the hydrogelators and corresponding hydrogels, the data is discussed first with respect to the evaluation of linear dichroism/birefringence, followed by the homogeneity of the sample, comparison of the effect of the differing light source (conventional versus SR) and, finally, thermal reversibility (gel-sol-gel).

\section{Linear dichroism/birefringence evaluation for SRCD}

Due to the small cross-sectional area of the beam (thus decreased averaging of sample compared to conventional CD) and the potential for alignment in the sample due to self-assembly, it was necessary to ascertain if any linear dichroism (LD) or birefringence was present. After visual inspection of the samples, the first SRCD experiment was therefore to evaluate the presence of any optical artefacts. To achieve this, the spectrum of the sample "spot 2" was acquired at two different orientations $\left(0^{\circ}\right.$ and $90^{\circ}$ ), which was facilitated by a rotating platform in the sample compartment of Module A (Figure 3).

For the hydrogels A-C of the sugar-containing hydrogelator $\mathbf{1}$, no significant LD or birefringence features were observed after the SRCD spectra were recorded at this single spot in the two orientations (Figure 4 (i)-(iii)). Hydrogel $\mathbf{A}$ in water showed identical SRCD spectra in both orientations, demonstrating the usefulness of the method. For hydrogel $\mathbf{A}$ in water, which is orientation independent (Figure 4 (i)), the SRCD data therefore indicated negligible artefacts and any spectral differences between the spots will indicate solely differences in chiral architecture. For hydrogels $\mathbf{B}$ and $\mathbf{C}$ in PBS, however, as the SRCD spectra were orientation dependent (Figure 4 (ii) and 4 (iii)), it was observed that chirality and/or optical artefacts were different upon rotation of the sample. The multiplication of the spectra by a scaling factor to obtain very similar spectra indicated differences in sample concentration in the matrix, as the thickness should remain constant inside the cell pathlength. Although the sample concentration of the same "spot" on rotation should be consistent, minor difference are possible due to the beam shape being ellipsoidal rather than spherical. Therefore, the SRCD spectra of hydrogels $\mathbf{A}-\mathbf{C}$, as a function of rotation normal to the propagation of incident light $\left(0^{\circ}\right.$ and $\left.90^{\circ}\right)$, indicated no LD and LB artefacts.

In contrast, the CD spectrum of hydrogel $\mathbf{D}$ (from $\mathbf{2}$ when thermally triggered in water) was much weaker in intensity and had a poor signal-to-noise ratio (Figure 4 (iv)). This was unsurprising as, of the two sugar-based hydrogelators, GlcNHFmoc 2 gives the weaker hydrogels based on gravitational tube inversion method. Therefore a lesser extent of assembly is observed for $\mathbf{2}$ under the gelation conditions employed relative to GalNHFmoc 1 and thus the weak CD signal observed. This observation for GlcNHFmoc 2 and GalNHFmoc 1 was in contrast to that published previously, where rheology confirmed that GlcNHFmoc 2 was mechanically stiffer than the GalNHFmoc $1,{ }^{17}$ however a different gelation procedure was utilised. Gelation is a dynamic process which is affected by both thermodynamic and kinetic processes and any modifications to gelation procedure can result in marked differences in macroscopic behaviour. Therefore, this apparent contradiction in macroscopic behaviour is typical of the nature of self-assembly process for gelation. Therefore, only one of the four conditions to form hydrogels of $\mathbf{2}$ was studied (Table 1). The hydrogel $\mathbf{E}$ (from Fmoc-FF $\mathbf{3}$ when triggered by sonication in PBS) had superimposable spectra obtained at two orientations (Figure $4(\mathrm{v}))$. The spectra of hydrogel $\mathbf{E}$ were consistent with previously reported CD spectra for hydrogels formed from $\mathbf{3}$ via a different gelation method. ${ }^{32,35}$ Therefore, with the exception of hydrogel $\mathbf{D}$ (from 2, a very soft dynamic gel), the hydrogels had no significant $\mathrm{CD}$ artefacts. Of the three hydrogels formed from 1, it is of note that the CD intensity of hydrogel $\mathbf{C}$ (gelled in PBS, thermally) is greatly reduced by comparison to hydrogel $\mathbf{A}$ (gelled in water thermally) and hydrogel $\mathbf{B}$ (gelled in PBS by sonication). The CD intensity is consistent with observations of the gel stiffness by gravitational tube inversion and is presumably due to alteration of the self-assembly under these conditions.

\section{INSERT FIGURE FOUR HERE}

FIGURE 4 Evaluation of linear dichroism and birefringence artefacts by comparison of SRCD spectra obtained at two orientations $\left(0^{\circ}\right.$ and $\left.90^{\circ}\right)$. (i) GalNHFmoc hydrogel A in water triggered thermally $(0.1 \mathrm{~mm}$ PL cell); (ii) GalNHFmoc hydrogel B in PBS triggered by sonication (0.2 mm PL cell); (iii) GalNHFmoc hydrogel $\mathbf{C}$ in PBS triggered thermally $(0.1 \mathrm{~mm}$ PL cell); (iv) GlcNHFmoc hydrogel $\mathbf{D}$ in water (triggered thermally ( $0.2 \mathrm{~mm}$ PL cell); (v) Fmoc-FF hydrogel E in PBS triggered by sonication $(0.1 \mathrm{~mm} \mathrm{PL}$ cell). All samples were gelled in the cell of the path length $(P L)$ stated at a concentration of $2.0 \mathrm{mg} / \mathrm{mL}$. 


\section{Chiral homogeneity of hydrogels}

SRCD spectra of the hydrogel specimens showed very similar homogeneity across spots 1-3 (as indicated in Figure 3). The general trend, with the exception of hydrogel D formed from 2 (Figure 5 (iv)), was that the first two sampling sites (spots 1 and 2) gave comparable spectra, whereas the third sampling site (spot 3) gave a different spectrum (Figure 5 (i)-(iii)). It is noted that the difference between the spots 1 and 2 versus spot 3 for hydrogel E (from 3) appears to be consistent with a matrix concentration difference (Figure $5(\mathrm{v})$ ).

For the GalNHFmoc hydrogel $\mathbf{A}$ in $\mathrm{H}_{2} \mathrm{O}$, the SRCD spectra of spot 1 and 2 were identical, indicating that that area of the hydrogel has the same gel matrix topology (Figure 5 (i)). The spectrum of spot 3 differs slightly quantitatively after the spectrum is offset to superimpose in the 290-310 nm range with the other two spots. However, it is qualitatively quite similar to the other two spots, indicating good homogeneity. For the GalNHFmoc hydrogel B in PBS, only spot 1 and 2 were identical (Figure 5 (ii)). However, the spectrum of spot 3 was rather distorted around $260 \mathrm{~nm}$. For the GalNHFmoc hydrogel $\mathbf{C}$ in PBS, only spot 1 and 2 were very similar (Figure 5 (iii)). Spot 3 was distorted in a similar manner to that of GalNHFmoc hydrogel B. The PMT (HV) data recorded confirmed that, for spot 3 for hydrogels $\mathbf{B}$ and $\mathbf{C}$, the sampling was too close to the cell wall resulting in masking of the beamlight (see Figure $\mathrm{C}$ of $\mathrm{SI}$ ). This was not the case for spot 3 of hydrogels $\mathbf{A}$, D and $\mathbf{E}$ (Figure 5(i), (iv) and (v)). Therefore no further interpretation of spot 3 in Figure 5(ii) and 5(iii) was undertaken. For the GlcNHFmoc hydrogel $\mathbf{D}$ in $\mathrm{H}_{2} \mathrm{O}$, the spectra were only qualitatively similar in the near UV region (Figure 5 (iv)). For the Fmoc-FF hydrogel $\mathbf{E}$ in PBS, the SRCD spectra of spot 1 and 2 were identical, indicating that that area of the hydrogel had the same gel matrix topology (Figure $5(\mathrm{v})$ ). Spot 3, after offset, gave a similar spectrum to the other 2 spots.

The shape, volume and material of the vessel in which the hydrogel is formed can alter the features of the hydrogel matrix topology. This is, in part, due to the dynamic nature of the selfassembly, which can be altered by local features, such as the wall of the cell. The altered behaviour of the hydrogel near the wall of the vessel is colloquially described as a "wall effect". However, it is not feasible to study the wall separately to the bulk of the hydrogel by standard characterization techniques.

The manual nature of sampling limited precise mapping of the spots but, practically, spot 3 was closer to the cell wall hence why the masking occurred for spot 3 for hydrogels $\mathbf{B}$ and $\mathbf{C}$. Since the PMT voltage was similar for all spots in hydrogels $\mathbf{A}, \mathbf{C}$ and $\mathbf{E}$, then their SRCD spectra indicate that where spot 3 had a different SRCD spectrum, this is becaude the sample was not homogeneous e.g. spot 3 may have a different hydrogel matrix realtive to spots 1 and 2 . This is the basis from which we postulate a 'wall effect'. To further explore this, the beamtime work should be revisited with an $X-Y$ mapping stage to more accurately 'map' the CD data approaching the cell wall. This will be the focus for further beamtime studies.

This demonstrates the power of the smaller cross section of the SRCD beamlight for such analysis. If the hydrogel and corresponding preparation method can be optimized to minimize/avoid such wall effects, then it will result in improved application, e.g. a consistent material across the full diameter of a cell culture well. This is of great significance, as changes to the hydrogel (such as its rheology) are known to be critical to cell culture. ${ }^{36,37}$ In addition, SRCD could be particularly advantageous for the study of spatially resolved multicomponent supramolecular hydrogels. ${ }^{38,39}$ The recent CD imaging (CDi) facility developed at B23 can now achieve spatial resolution of $200-250 \mu \mathrm{m}$.

In contrast to all the other hydrogels studied, hydrogel $\mathbf{D}$ (from 2 triggered thermally in water) showed no consistency between the SRCD spectra obtained from three sites (Figure 5 (iv)). Therefore, the difference between the galactose and glucose motifs in the sugar-containing hydrogelators is profound, with the galactose residues promoting a greater extent and ability to selfassemble in both water and PBS (Figure 5). This is presumably due to the differing extent of self-assembly in the hydrogels matrix topology, i.e. hydrogels from GalNHFmoc 1 have a greater extent of self-assembly than those of GlcNHFmoc 2, which is evidenced by the $C D$ intensity.

\section{INSERT FIGURE FIVE HERE}

FIGURE 5 Comparison of SRCD spectra obtained from different sites/spots in a hydrogel sample. (i) GalNHFmoc hydrogel A in water triggered thermally $(0.1 \mathrm{~mm}$ PL cell); (ii) GalNHFmoc hydrogel $\mathbf{B}$ in PBS triggered by sonication ( $0.2 \mathrm{~mm}$ PL cell); (iii) GalNHFmoc hydrogel $\mathbf{C}$ in PBS triggered thermally $(0.1 \mathrm{~mm} \mathrm{PL}$ cell); (iv) GlcNHFmoc hydrogel $\mathbf{D}$ in water triggered thermally $(0.2 \mathrm{~mm} \mathrm{PL}$ cell); (v) Fmoc-FF hydrogel $\mathbf{E}$ in PBS triggered by sonication $(0.1 \mathrm{~mm}$ $\mathrm{PL}$ cell). All samples were gelled in the cell of the path length $(\mathrm{PL})$ stated at a concentration of $2.0 \mathrm{mg} / \mathrm{mL}$.

\section{Comparison of CD and SRCD spectra}

The homogeneity of the hydrogel within the cell can also be evaluated by comparison of the CD spectrum of a small area of the hydrogel by SRCD with that of a larger area of hydrogel by conventional CD. This is due to the different cross-sectional beamlight dimensions e.g. Chirascan on average has a $16 \mathrm{~mm}$ diameter area whereas SRCD has $0.5 \mathrm{~mm}$ diameter area. The assumption is that SRCD and CD spectra showing similar spectral features in terms of intensity and sign will be consistent with similar hydrogel matrix homogeneity. The SRCD and CD of the hydrogels appear very similar in terms of spectral features and relative intensity magnitude, indicating overall uniform and homogeneous hydrogel sample preparations (Figure 6). The absorbance spectra were also qualitatively similar (see Figure D of $\mathrm{SI}$ ). The greatest difference between $\mathrm{CD}$ and SRCD was observed for hydrogel C (from $\mathbf{1}$ in PBS triggered thermally, Figure 6 (iii)) and hydrogel $\mathbf{D}$ (from $\mathbf{2}$ in water triggered thermally, Figure 6(iv))

\section{INSERT FIGURE SIX HERE}

FIGURE 6 Comparison of CD (Chirascan, ECD) and SRCD spectral differences arising from differing cross sectional areas of the beam. (i) GalNHFmoc hydrogel $\mathbf{A}$ in water triggered thermally $(0.1 \mathrm{~mm} \mathrm{PL}$ cell); (ii) GalNHFmoc hydrogel B in PBS triggered by sonication (0.2 $\mathrm{mm}$ PL cell); (iii) GalNHFmoc hydrogel $\mathbf{C}$ in PBS triggered thermally (0.1 $\mathrm{mm}$ PL cell); (iv) GlcNHFmoc hydrogel $\mathbf{D}$ in water triggered thermally ( $0.2 \mathrm{~mm}$ PL cell); (v) Fmoc-FF hydrogel $\mathbf{E}$ in PBS triggered by sonication $(0.1 \mathrm{~mm} P L$ cell). All samples were gelled in the cell of the path length $(\mathrm{PL})$ stated at a concentration of $2.0 \mathrm{mg} / \mathrm{mL}$. All given SRCD spectra are of spot 2 at $0^{\circ}$ orientation. The corresponding absorbance spectra are provided in Figure D of the SI.

\section{Thermal studies}

The self-assembled hydrogels (A, B and E) were studied as a function of temperature by obtaining a spectrum after heating at $85^{\circ} \mathrm{C}$ and then again at room temperature to assess thermal reversibility. The UV absorbance spectrum $(215-300 \mathrm{~nm})$ of the gel should be similar to the "broken gel" (due to thermal disruption) with no major intensity changes. If this is the case, then the collapse of the CD spectrum indicates the presence of cancellation, either due to opposite chirality or different sample orientation, with the electronic transitions perpendicular to the direction of the propagation of incident light. The spectrum becomes like an oriented $C D$ spectrum compared to its $C D$ spectrum in solution. The heating of the hydrogel is referred to as thermal disruption as, upon heating, the non-covalent interactions which give rise to the matrix of the gel are broken, 
thus disrupting the gel network. Practically, as illustrated for hydrogel $\mathbf{A}$, it is found that the $C D$ spectrum of the hydrogel collapses on heating in a similar way to that observed for the solution spectrum, where the spectral feautres are lost (Figure 7, left). ${ }^{17}$ However, the absorbance spectra increase in intensity for the thermally destroyed gel and the solution when compared to the hydrogel (Figure 7, right). It is reasonable to assume, for the thermally disrupted gel, that at elevated temperature the solubility of the hydrogelator is enhanced increasing its concentration in solution.

\section{INSERT FIGURE SEVEN HERE}

FIGURE 7 CD (left) and absorbance (right) spectra of GalNHFmoc 1 as a $\mathrm{MeOH}$ solution and as a hydrogel before and after heating in a cylindrical non-demountable cell (PL $0.1 \mathrm{~mm})$ : (i) solution in $\mathrm{MeOH}$ (3.0 mg/mL); (ii) hydrogel $\mathbf{A}$ in water $(2.0 \mathrm{mg} / \mathrm{mL})$, thermally triggered - initial gel; (iii) hydrogel $\mathbf{A}$ in water $(2.0 \mathrm{mg} / \mathrm{mL})$, thermally triggered - after thermal destruction.

Given that the sol-gel process is reversible, it can be cycled to (i) confirm that the CD signal arises from the self-assembly, and (ii) explore the extent of self-assembly that can occur when the hydrogel is reformed. Hydrogels (A, B and $\mathbf{E})$ were heated and their spectra acquired by $C D$, then recorded again after the hydrogel was allowed to reform (Figure 8). It was found that monitoring the reformation of the hydrogel was best by $C D$ (rather than SRCD) due to the different cross-sectional beamlight dimensions. The sugar-containing hydrogels analysed were able to revert to a negligible CD signal after 30 minutes of heating (Figure 8 (i) and 8 (ii)) and were therefore comparable to their solution spectrum (see Figures $A$ and $B$ of $\mathrm{SI}$ ). However, the hydrogel of Fmoc-FF 3 (E) did not revert to a negligible CD spectrum after this time (Figure 8 (iii)). Comparison to the corresponding methanolic solution of $\mathbf{3}$, which does give a negligible $C D$ spectrum (see Figure $A$ of $\mathrm{SI}$ ), suggests that selfassembly persists to some extent after the period of heating.

\section{INSERT FIGURE EIGHT HERE}

FIGURE 8 Thermal studies of hydrogels to ascertain the contribution of the self-assembly to the $C D$ spectrum and the reversibility of gelation. (i) GalNHFmoc hydrogel A in water triggered thermally ( 0.1 $\mathrm{mm}$ PL cell); (ii) GalNHFmoc hydrogel $\mathbf{B}$ in PBS triggered by sonication (0.2 mm PL cell); (iii) Fmoc-FF hydrogel $\mathbf{E}$ in PBS triggered by sonication $(0.1 \mathrm{~mm}$ PL cell). All samples were gelled in the cell of the path length $(\mathrm{PL})$ stated at a concentration of $2.0 \mathrm{mg} / \mathrm{mL}$. The corresponding absorbance spectra are provided in Figure $\mathrm{E}$ of the SI.

Further to this, several samples were found to have altered CD intensities for their gels after the gel-sol-gel process, where the hydrogels either decreased (A and E, Figure 8 (i) and 8 (iii)) or increased ( $\mathbf{B}$, Figure 8 (ii)) intensity by CD. It is of note that the process of thermal disruption of the gels shown in Figure 8 was different to that of the initial gelation method e.g. the heating/cooling rate differed for hydrogel $\mathbf{A}$ whereas hydrogels $\mathbf{B}$ and $\mathbf{E}$ were formed by sonication only. It is widely reported that differences in gelation conditions can have a profound effect on resulting gelation. ${ }^{40} \mathrm{An}$ increased intensity (assuming no change has occurred to the self-assembly mechanism) would suggest that either (i) the hydrogel had not been fully self-assembled within the cell after the first sol-gel cycle and/or (ii) the extent of self-assembly increased after a second sol-gel cycle. It is clear that the hydrogels formed by sugar-containing structures (1 and
2), which are devoid of the $-\mathrm{COOH}$ moiety, have very different behaviour compared to that of dipeptide $\mathbf{3}$. This is presumably due to the lack of ionic interactions, the ability of the sugar motif to mutarotate and the ability of hydrogels of 1 and $\mathbf{2}$ (A-D) to form $\mathrm{CH}-\pi$ interactions (as previously evidenced for GalNHFmoc 1 and GlcNHFmoc 2). ${ }^{17}$

\section{Discussion}

For most compounds, analysis of the SRCD data showed no significant contribution from linear dichroism or birefringence. The hydrogel of GlcNHFmoc 2 was the exception to this which was presumably due to its softer nature (which was observed by the tube inversion method). Comparison between CD and SRCD spectra for each hydrogel sample also gave further information with regard to homogeneity. The CD spectra were generally higher in intensity than the SRCD spectra, which could be interpreted as being due to a greater extent of self-assembly relative to the area sampled. This study demonstrates that the nature and extent of chiral homogeneity of hydrogel materials was better assessed with the combined use of SRCD multi-site sampling and the larger sampling area of benchtop $C D$ instruments. Thermal studies conducted using CD identified that (i) the self-assembly of the Fmoc-FF hydrogel (E) was not fully disrupted after 30 minutes of heating and (ii) different samples had altered CD intensities after completion of sol-gel cycling. These observations will inform preparation and thus guide application of these materials. The Fmoc-peptide-based hydrogel $\mathbf{E}$ showed differences in chiral homogeneity compared to the hydrogels A-D of the sugar-based hydrogelators. This is in part due to its ionic nature, which arises from the $-\mathrm{COOH}$ motif and, thus, a different mode of assembly. It is clear that the different hydrogelators have different tolerances for gelation triggers, as well as their corresponding environments, with GalNHFmoc 1 being the more robust and versatile hydrogelator. This can be shown clearly for GalNHFmoc 1, since it can form a hydrogel in both PBS and water, as well as by both sonication and thermal triggers (Figure 9). Although the corresponding spectra are qualitatively similar (i.e. the same two positive $C D$ bands ca. 220 and $270 \mathrm{~nm}$ ), the GalNHFmoc hydrogel B in PBS triggered by sonication is slightly red shifted and has an altered absorbance profile. These differences in spectral features suggest that there may be differences in the corresponding mode of self-assembly for different gelation methods. Such differences in gelation behaviour can therefore be introduced by relatively simple modifications to (i) structure, as $\mathbf{1}$ and $\mathbf{2}$ only differ in stereochemistry at one chiral centre, and (ii) the gelation method, as evidenced for hydrogels of 1 (Figures 8 (i), 8 (ii), 9).

\section{INSERT FIGURE NINE HERE}

FIGURE 9 CD (left) and absorbance (right) spectra of hydrogels of GalNHFmoc 1 prepared by different conditions. (Gel A) GalNHFmoc hydrogel in water triggered thermally $(0.1 \mathrm{~mm} \mathrm{PL}$ cell); (Gel B) GalNHFmoc hydrogel in PBS triggered by sonication $(0.2 \mathrm{~mm} \mathrm{PL}$ cell); (Gel C) GalNHFmoc hydrogel in PBS triggered thermally (0.1 $\mathrm{mm} \mathrm{PL}$ cell). All samples were gelled in the cell of the path length $(\mathrm{PL})$ stated at a concentration of $2.0 \mathrm{mg} / \mathrm{mL}$.

\section{CONCLUSION}


The results and discussion presented herein explores how the combined use of benchtop/commercial CD and SRCD can be used to obtain a wealth of information about LMW hydrogels as long as suitable sample optimisation is undertaken and a series of experiments are planned in a cohesive manner. A key advantage of SRCD was the small cross-section of the beamlight that allowed for multi-site sampling of the gel film to assess its homogeneity in terms of chirality and thickness. The temperature study also showed that the gelation process was also reversible. Due to the large crosssection of the beamlight generated with benchtop CD instruments $(8 \mathrm{~mm} \times 10 \mathrm{~mm})$, the CD spectrum of a hydrogel is the average chirality of the whole area irradiated by the incident light. The use of SRCD, on the other hand, enables the assessment of the homogeneity in terms of chirality of the hydrogel sample as the area can be sampled at much smaller spatial resolution. In this study, the cross-section of the beamlight of B23 beamline was about $250 \mu \mathrm{m}$ and also highly collimated relative to benchtop CD instruments and other SRCD worldwide beamlines. In summary, the combined use of $C D$ and SRCD has revealed that several of the prepared hydrogels were not homogeneous in terms of chirality and thickness and highlights the differences that can occur in gelation from modification of gelation procedure i.e. the thermal disruption and reformation of the gels. Therefore, this method can be used successfully for quality control to assess the best method of gel preparation to obtain the required specification and properties.

\section{Supporting information}

Additional supporting information may be found in the online version of this article at the publisher's website and includes a detailed description of the approach for optimisation of sample preparation to obtain the spectra, the absorbance data that corresponds to Figures 6 and 8, and the solution CD and absorbance spectra of hydrogelators 1-3 in methanol.

\section{REFERENCES AND NOTES}

1. Webber MJ, Appel EA, Meijer EW, Langer R. Supramolecular Biomaterials. Nat Mater. 2015;15(1):13-26.

2. Gao Y, Zhao F, Wang Q, Zhang Y, Xu B. Small Peptide

Nanofibers as the Matrices of Molecular Hydrogels for Mimicking

Enzymes and Enhancing the Activity of Enzymes. Chem Soc

Rev. 2010;39(9):3425-3433.

3. Boynuegri TA, Gürü M. Catalytic Dehydrogenation of Calcium Borohydride by Using Hydrogel Catalyst. Int J Hydrogen Energy. 2017;42(28):17869-17873.

4. Zhao X, Pan F, Xu H, Yaseen M, Shan H, Hauser CaE, Zhang

S, Lu JR. Molecular Self-Assembly and Applications of Designer Peptide Amphiphiles. Chem Soc Rev. 2010;39(9):3480-3498.

5. Ikeda M, Ochi R, Wada A, Hamachi I. Supramolecular Hydrogel Capsule Showing Prostate Specific Antigen-Responsive Function for Sensing and Targeting Prostate Cancer Cells. Chem Sci. 2010;1(4):491-498.

6. O'Brien FJ. Biomaterials \& Scaffolds for Tissue Engineering. Mater Today. 2011;14(3):88-95.

7. Ryan DM, Nilsson BL. Self-Assembled Amino Acids and Dipeptides as Noncovalent Hydrogels for Tissue Engineering. Polym Chem. 2012;3(1):18-33.

8. Tan H, Marra KG. Injectable, Biodegradable Hydrogels for Tissue Engineering Applications. Materials. 2010; 3(3):1746-1767. 9. Caliari SR, Burdick JA. A Practical Guide to Hydrogels for Cell Culture. Nat Methods. 2016; 13(5):405-414.

10. Jayawarna V, Richardson SM, Hirst AR, Hodson NW, Saiani A, Gough JE, Ulijn RV. Introducing Chemical Functionality in Fmoc-Peptide Gels for Cell Culture. Acta Biomater.

2009;5(3):934-943.
11. Liebmann T, Rydholm S, Akpe V, Brismar H. Self-Assembling Fmoc Dipeptide Hydrogel for in Situ 3D Cell Culturing. BMC Biotechnol. 2007;7(1):88:1-11.

12. Yang Z, Liang G, Ma M, Abbah AS, Lu WW, Xu B. D Glucosamine-Based Supramolecular Hydrogels to Improve Wound Healing. Chem Commun. 2007;8:843-845.

13. Agubata C O, Okereke C, Nzekwe IT, Onoja RI, Obitte NC. Development and Evaluation of Wound Healing Hydrogels Based on a Quinolone, Hydroxypropyl Methylcellulose and Biodegradable Microfibres. Eur J Pharm Sci. 2016:89:1-10. 14. Eskandari S, Guerin T, Toth I, Stephenson RJ. Recent Advances in Self-Assembled Peptides: Implications for Targeted Drug Delivery and Vaccine Engineering. Adv Drug Deliv Rev. 2016;(110-111):169-187.

15. Vashist A, Vashist A, Gupta YK, Ahmad S. Recent Advances in Hydrogel Based Drug Delivery Systems for the Human Body. $J$ Mater Chem B. 2014;2(2):147-166.

16. Skilling KJ, Kellam B, Ashford M, Bradshaw TD, Marlow M. Developing a Self-Healing Supramolecular Nucleoside Hydrogel. Soft Matter. 2016;12(43):8950-8957.

17. Birchall LS, Roy S, Jayawarna V, Hughes M, Irvine E, Okorogheye GT, Saudi N, De Santis E, Tuttle T, Edwards AA, Uliyn RV Exploiting $\mathrm{CH}-\pi$ Interactions in Supramolecular Hydrogels of Aromatic Carbohydrate Amphiphiles. Chem Sci. 2011;2(7):1349-1355

18. Takahashi O, Kohno Y, Nishio M. Relevance of Weak Hydrogen Bonds in the Conformation of Organic Compounds and Bioconjugates: Evidence from Recent Experimental Data and High-Level Ab Initio MO Calculations. Chem Rev.

2010;110(10):6049-6076.

19. Raeburn J, Zamith Cardoso A, Adams DJ. The Importance of the Self-Assembly Process to Control Mechanical Properties of Low Molecular Weight Hydrogels. Chem Soc Rev. 2013;42(12):5143-5156.

20. Du X, Zhou J, Shi J, Xu B. Supramolecular Hydrogelators and Hydrogels: From Soft Matter to Molecular Biomaterials. Chem Rev. 2015;115(24):13165-13307.

21. Liu J, Sun Z, Yuan Y, Tian X, Liu X, Duan G, Yang Y, Yuan L., Lin HC, Li X. Peptide Glycosylation Generates Supramolecular Assemblies from Glycopeptides as Biomimetic Scaffolds for Cell Adhesion and Proliferation. ACS Appl Mater Interfaces.

2016;8(11):6917-6924.

22. Yoza K, Amanokura N, Ono Y, Akao T, Shinmori H, Takeuchi M, Reinhoudt DN. Sugar-Integrated Gelators of Organic Solvents Their Remarkable Diversity in Gelation Ability and Aggregate Structure. Chem Eur J. 1999;5(9):2722-2729.

23. Jung JH, Rim JA, Han WS, Lee SJ, Lee YJ, Cho EJ, Kim JS, Ji Q, Shimizu T. Hydrogel Behavior of a Sugar-Based Gelator by Introduction of an Unsaturated Moiety as a Hydrophobic Group. Org Biomol Chem. 2006;4:2033-2038.

24. Smith DK. Lost in translation? Chirality effects in the selfassembly

of nanostructured gel-phase materials. Chem Soc Rev. 2009;38:684-694.

25. Wang $\mathrm{Y}, \mathrm{Xu} \mathrm{J}$, Wang $\mathrm{Y}$, Chen $\mathrm{H}$. Emerging chirality in nanoscience.

Chem Soc Rev. 2013;42:2930-2962.

26. Amdursky N, Stevens MM. Circular Dichroism of Amino Acids: Following the Structural Formation of Phenylalanine. Chem Phys Chem. 2015;16(13):2768-2774.

27. Berova N, Di Bari L, Pescitelli G. Application of Electronic Circular Dichroism in Configurational and Conformational Analysis of Organic Compounds. Chem Soc Rev. 2007;36(6):914931.

28. Pescitelli G, Di Bari L, Berova N. Conformational Aspects in the Studies of Organic Compounds by Electronic Circular Dichroism. Chem Soc Rev. 2011;40(9):4603-4625.

29. Pescitelli G, Di Bari L, Berova N. Application of Electronic Circular Dichroism in the Study of Supramolecular Systems. Chem Soc Rev. 2014;43(15):5211-5233.

30. Yu G, Yan X, Han C, Huang F. Characterization of supramolecular

gels. Chem Soc Rev. 2013;42:6697-6722. 
31. Raeburn J, Pont G, Chen L, Cesbron Y, Lévy R, Adams DJ. Fmoc-Diphenylalanine Hydrogels: Understanding the Variability in Reported Mechanical Properties. Soft Matter. 2012;8(4): 11681174.

32. Smith AM, Williams RJ, Tang C, Coppo P, Collins RF, Turner ML, Saiani A, Ulijn RV. Fmoc-Diphenylalanine Self Assembles to a Hydrogel via a Novel Architecture Based on $\pi-\pi$ Interlocked $\beta$ Sheets. Adv Mater. 2008;20(1);37-41.

33. Hussain R, Jávorfi T, Siligardi G. Circular Dichroism Beamline B23 at the Diamond Light Source. J Synchrotron Radiat.

2012;19(1):132-135.

34. Hussain R, Jávorfi T, Rudd TR, Siligardi G. High-Throughput SRCD Using Multi-Well Plates and Its Applications. Sci Rep.

2016;6:38028:1-6.

35. Zhou M, Smith AM, Das AK, Hodson NW, Collins RF, Ulijn RV, Gough JE. Self-Assembled Peptide-Based Hydrogels as Scaffolds for Anchorage-Dependent Cells. Biomaterials. 2009;30(13):2523-2530
36. Yan C, Pochan DJ. Rheological Properties of Peptide-Based Hydrogels for Biomedical and Other Applications. Chem Soc Rev. 2010;39(9):3528-3540

37. Zuidema JM, Rivet CJ, Gilbert RJ, Morrison FA. A Protocol for Rheological Characterization of Hydrogels for Tissue Engineering

Strategies. J Biomed Mater Res - Part B Appl. Biomater. 2014; 102(5):1063-1073

38. Draper ER, Eden EGB, McDonald TO and Adams DJ. Spatially resolved multicomponent gels. Nature Chem. 2015;7:848-852.

39. Cornwell DJ, Oliver J. Daubney, and David K. Smith Photopatterned Multidomain Gels: Multi-Component SelfAssembled Hydrogels Based on Partially Self-Sorting 1,3:2,4Dibenzylidene-D-sorbitol Derivatives. J Am Chem Soc. 2015;137:15486-15492.

40.Raeburn J, Cardoso AZ, Adams DJ. The importance of the self-assembly process to control mechanical properties of low molecular weight hydrogels. Chem. Soc. Rev.2013;42;51435156.

\section{Graphical Abstract}
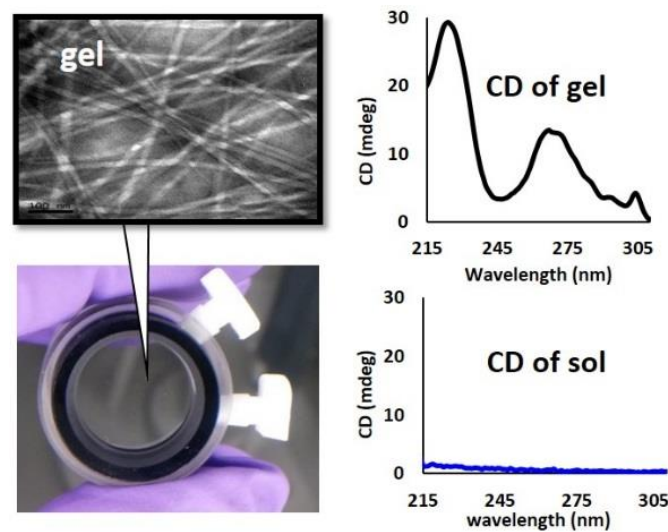

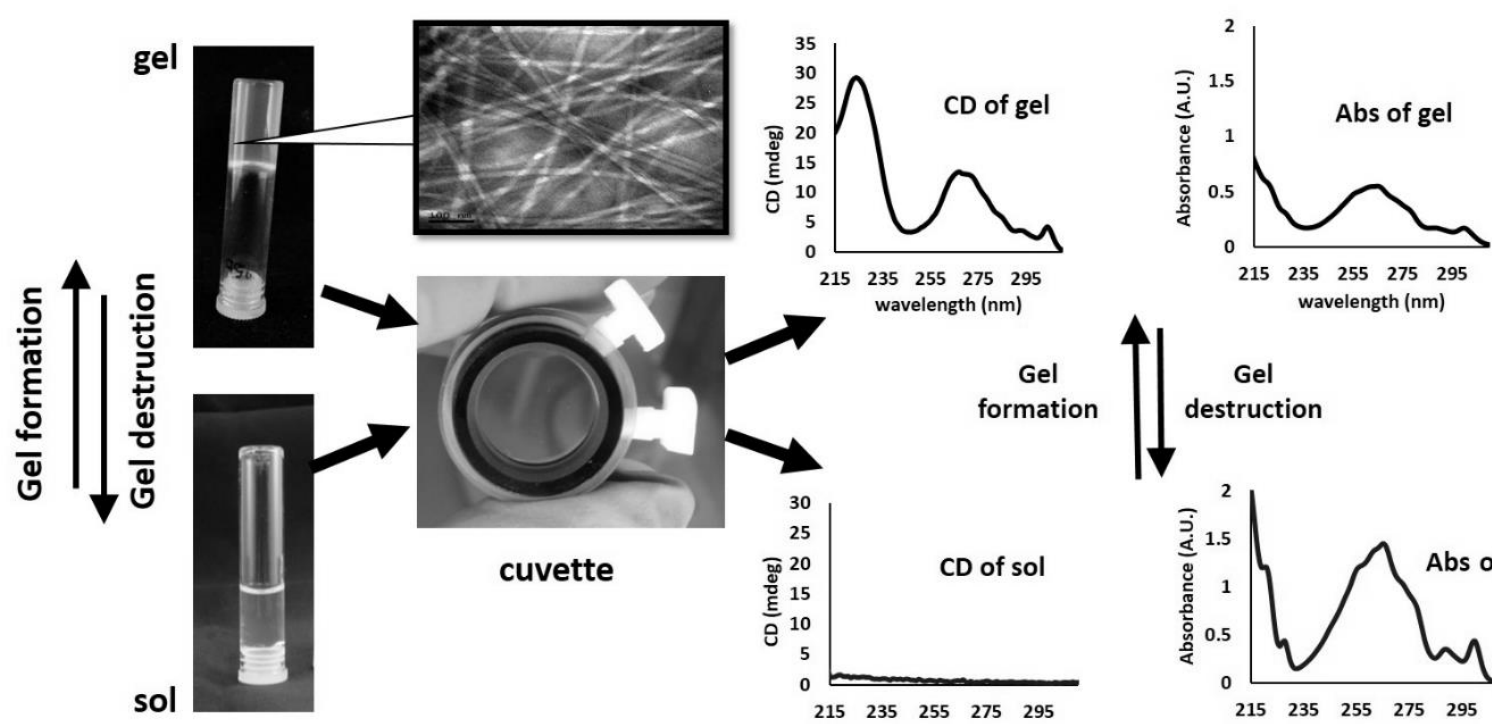

FIGURE 1
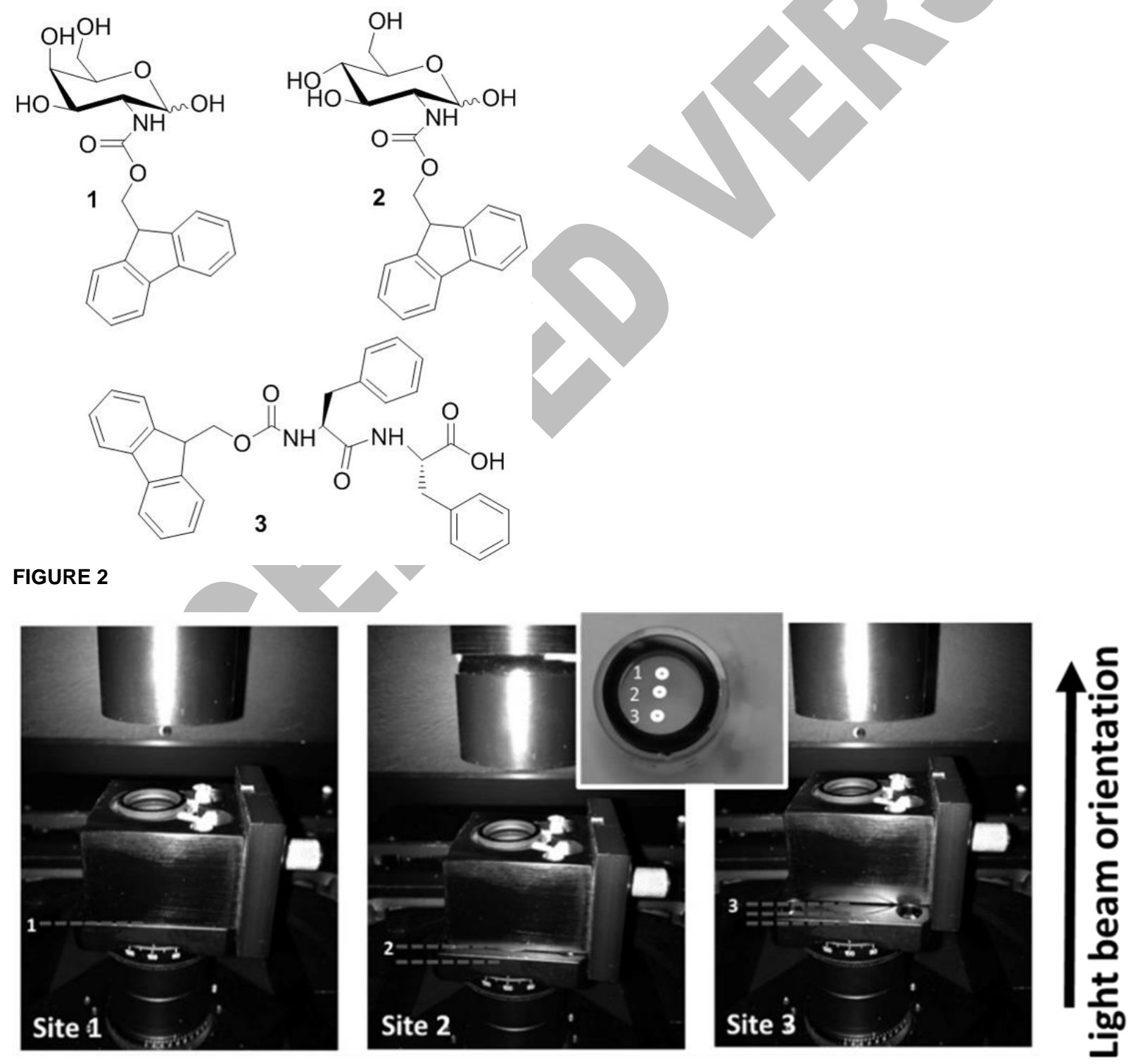

FIGURE 3 
TABLE 1 Gelation conditions. The concentration of all samples was $2.0 \mathrm{mg} / \mathrm{mL}$. (*The hydrogelator also gels under these conditions but was not included in this study.)

\begin{tabular}{|c|c|c|c|}
\hline Hydrogelator & Solvent & Method & Hydrogel \\
\hline \multirow{4}{*}{ GalNHFmoc 1 } & Water & Sonication & ${ }^{*}$ \\
\cline { 2 - 4 } & Water & Thermal & $\mathbf{A}$ \\
\cline { 2 - 4 } & PBS & Sonication & B \\
\cline { 2 - 4 } & PBS & Thermal & $\mathbf{C}$ \\
\hline \multirow{4}{*}{ GlcNHFmoc 2 } & Water & Sonication & ${ }^{*}$ \\
\cline { 2 - 4 } & Water & Thermal & D \\
\cline { 2 - 4 } & PBS & Sonication & ${ }^{*}$ \\
\cline { 2 - 4 } & PBS & Thermal & ${ }^{*}$ \\
\hline Fmoc-FF 3 & PBS & Sonication & E \\
\hline
\end{tabular}

\section{TABLE 1}
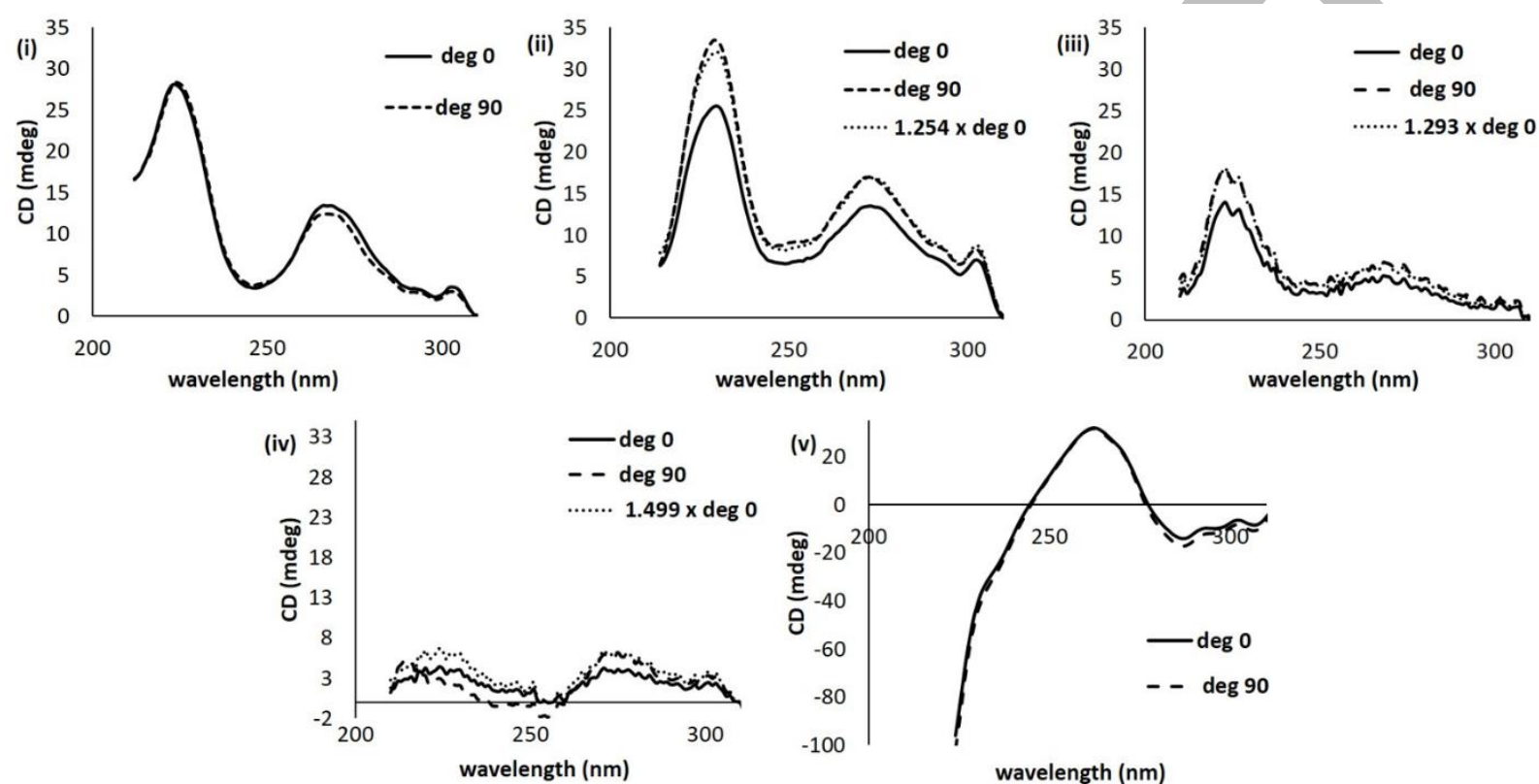

FIGURE 4
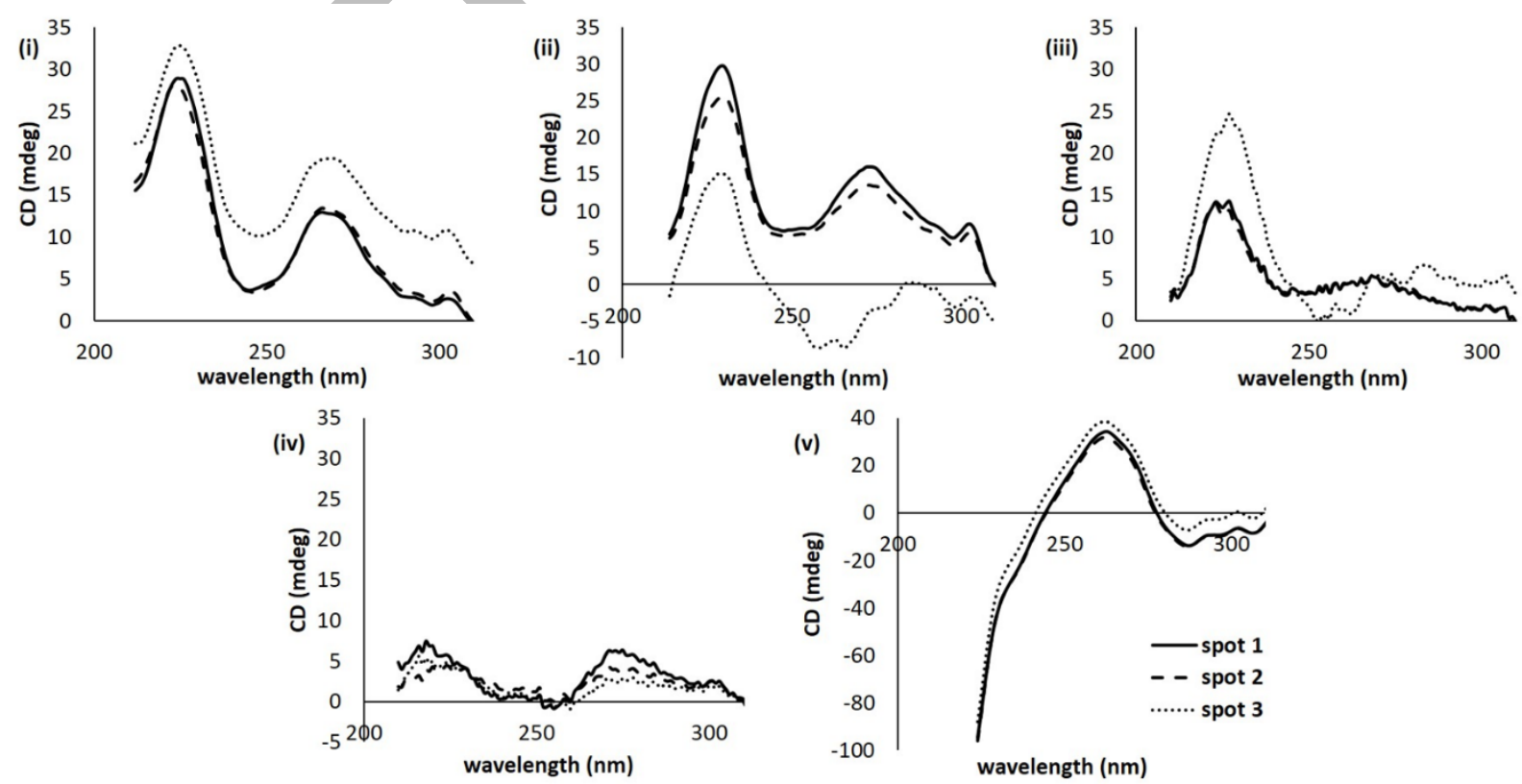

FIGURE 5 

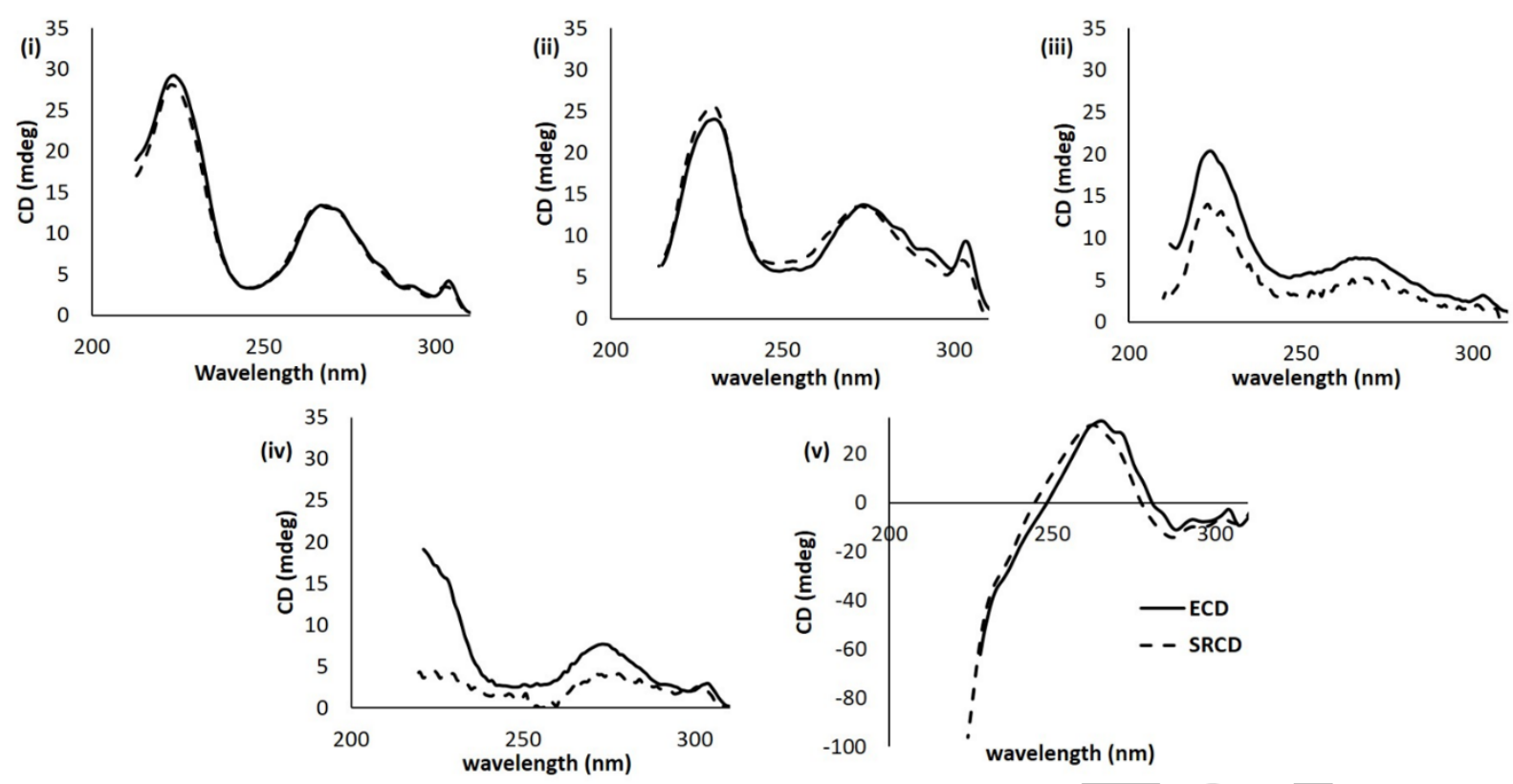

FIGURE 6
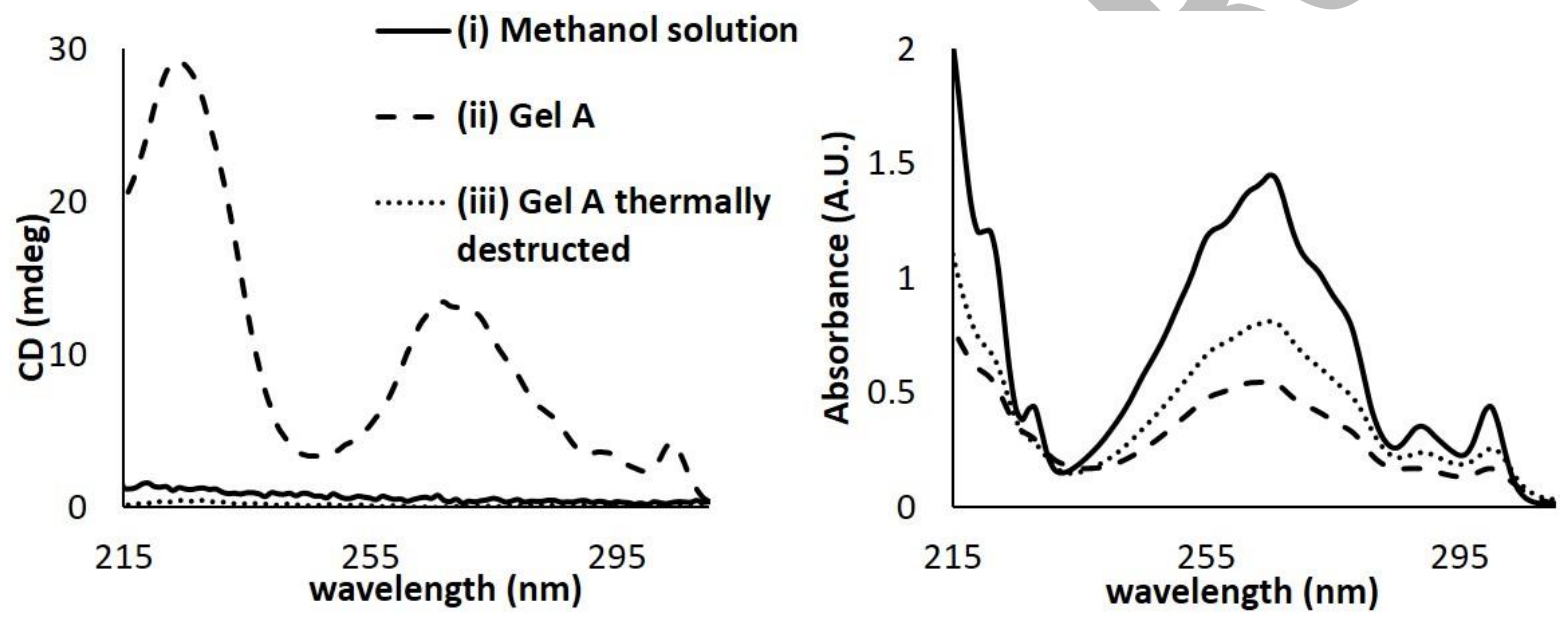

FIGURE 7
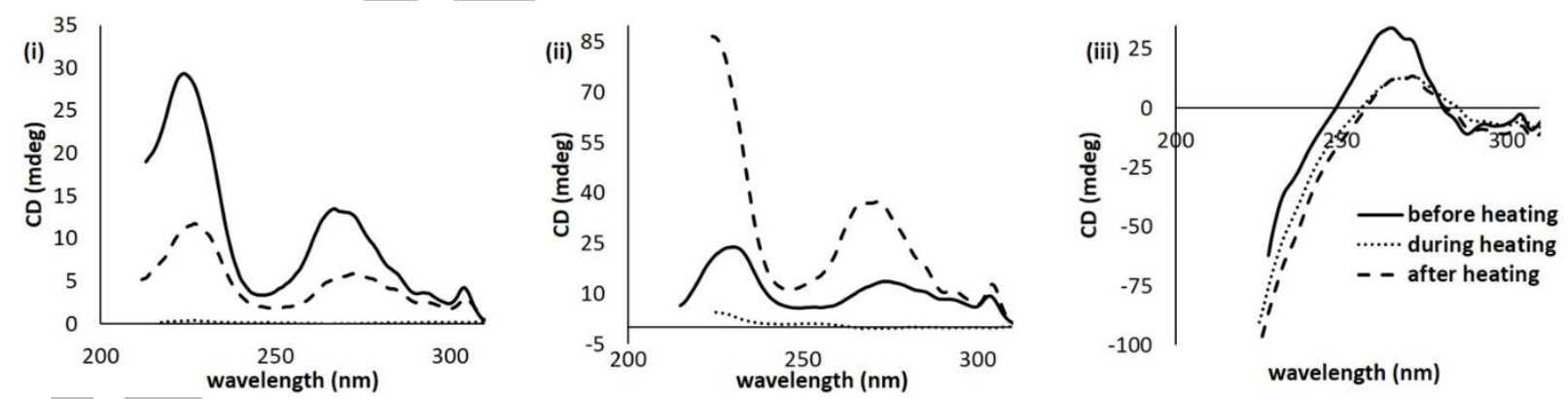

FIGURE 8 

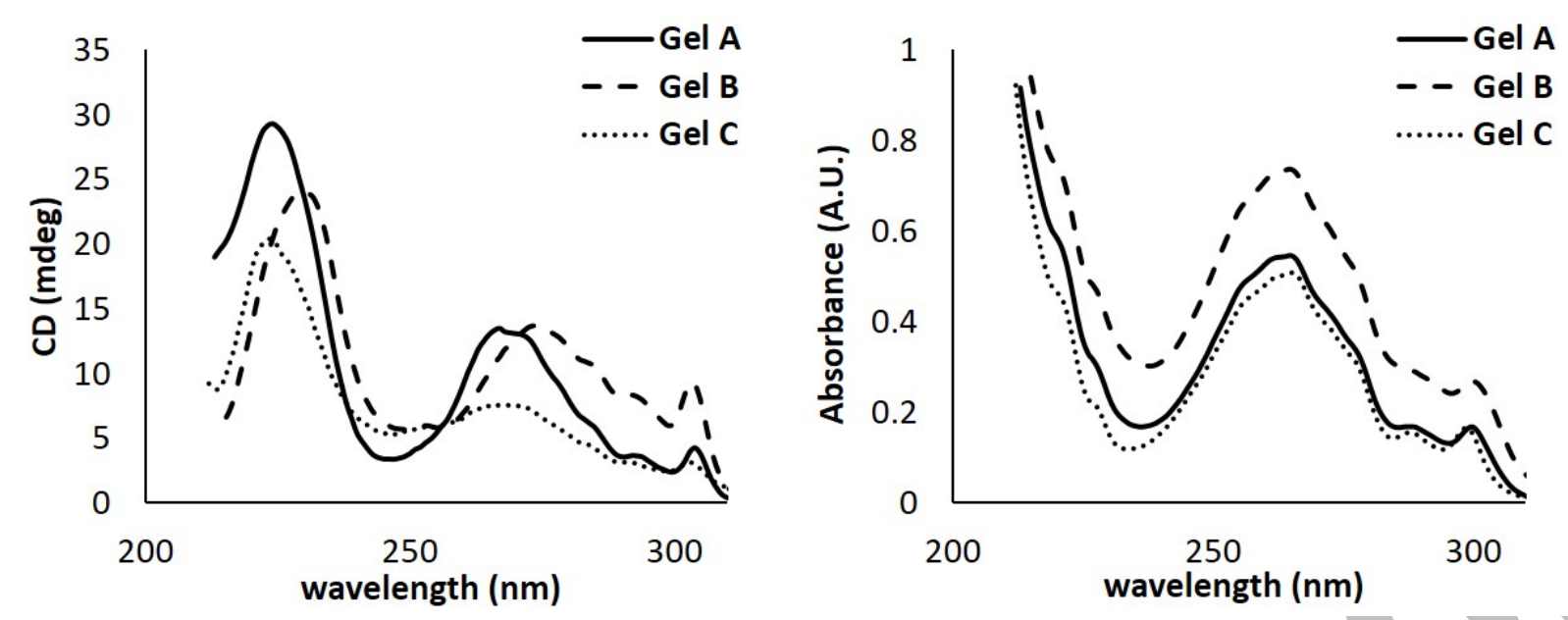

FIGURE 9 


\section{Supporting information for:}

\section{Circular dichroism studies of low molecular weight hydrogelators: addressing practical issues}

Efstratios D. Sitsanidis ${ }^{1} \mid$ Carmen C. Piras $^{1} \mid$ Bruce D. Alexander $^{2} \mid$ Giuliano Siligardi $^{3} \mid$ Tamas Javorfi ${ }^{3} \mid$ Andrew J. Hall ${ }^{1} \mid$ Alison A. Edwards*1

${ }^{1}$ Medway School of Pharmacy, Universities of Greenwich and Kent at Medway, Anson Building, Central Avenue, Chatham Maritime, Kent, ME4 4TB, UK

${ }^{2}$ University of Greenwich, Central Avenue, Chatham, Kent, ME4 4TB, UK

${ }^{3}$ Diamond Light Source Ltd., Didcot, Oxfordshire, OX11 0DE, UK

Correspondence

A. A. Edwards, Medway School of Pharmacy, Universities of Greenwich and Kent at Medway, Anson Building, Central Avenue, Chatham Maritime, Kent, ME4 4TB, UK. Email: A.A.Edwards@kent.ac.uk

\section{Contains:}

1. Solution spectra of hydrogelators 1-3 in methanol to facilitate interpretation of the thermal studies.

2. Optimisation of approach to obtain hydrogel within the spectroscopic cell.

3. The PMT (HV) plots for the SRCD spectra shown in Figure 5 of the manuscript.

4. The absorbance spectra for the SRCD and CD spectra shown in Figure 6 of the manuscript.

5. The absorbance spectra for the CD spectra shown in Figure 8 of the manuscript. 
1. Solution spectra of hydrogelators 1-3 in methanol to facilitate interpretation of the thermal studies.
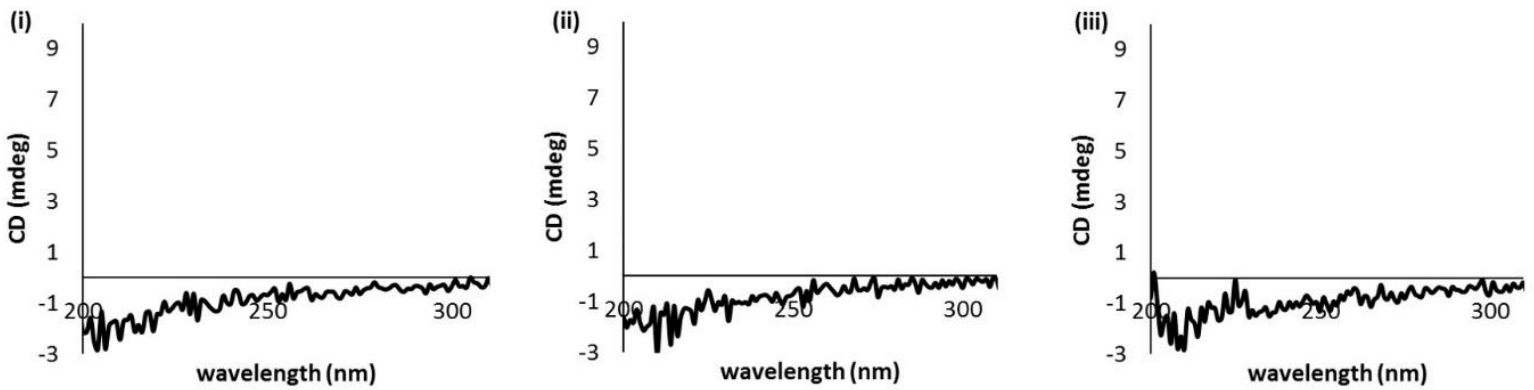

FIGURE A CD spectra of hydrogelators 1-3 in methanol. (i) GalNHFmoc 1; (ii) GlcNHFmoc 2; (iii) Fmoc-FF 3. All samples were recorded in a cylindrical non-demountable cell of the path length $0.1 \mathrm{~mm}$ at a concentration of $0.2 \mathrm{mg} / \mathrm{mL}$.
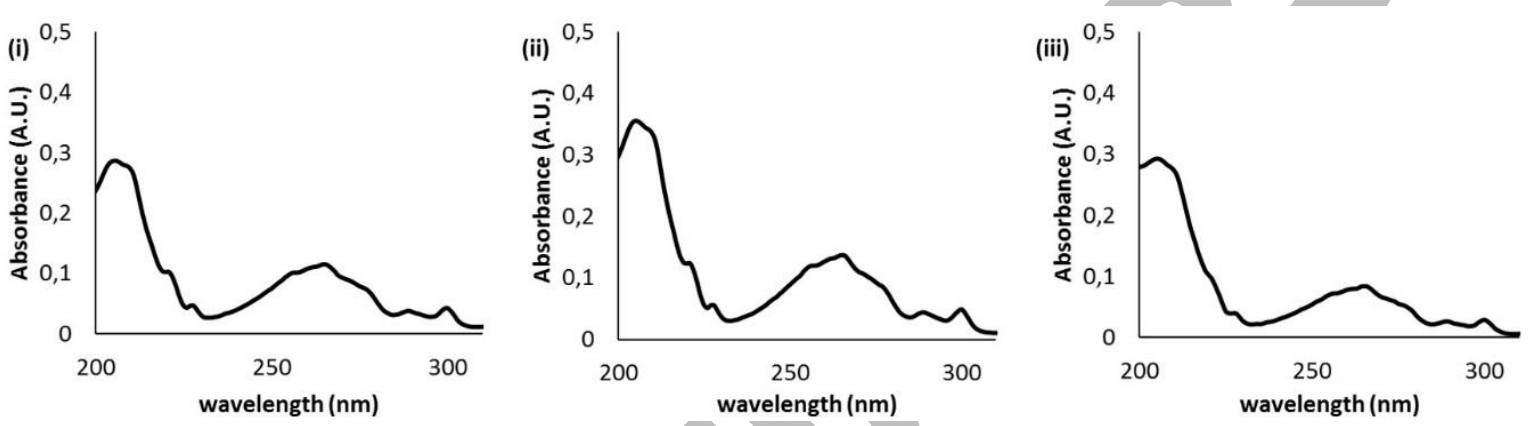

FIGURE B Absorbance spectra of hydrogelators 1-3 in methanol. (i) GalNHFmoc 1; (ii) GlcNHFmoc 2; (iii) Fmoc-FF 3. All samples were recorded in a cylindrical non-demountable cell of the path length $0.1 \mathrm{~mm}$ at a concentration of $0.2 \mathrm{mg} / \mathrm{mL}$. 


\section{Optimisation of approach to obtain hydrogel within the spectroscopic cell}

A number of approaches were employed to optimise gelation to obtain a hydrogel in a suitable spectroscopic cell. The success of each approach was evaluated by the intensity of the resulting conventional CD spectrum where a greater intensity indicated a greater extent of gelation obtained.

Preparation of the hydrogels in a vial with subsequent transfer to a demountable cell resulted in disruption of the hydrogel on transfer and further disruption on assembly of the cell (compression between cell faces) such that the gelation was not uniform throughout the cell. Similarly, transfer of the hydrogel (formed in a vial) by pipette into the cell also affected the resulting hydrogel in the cell. It was also found that when the hydrogelators were pre-treated (sonication and/or thermally) in a vial and then transferred as a solution to the cell, that the gelation was most reproducible in cylindrical cells rather than rectangular cells - presumably due to volume and/or shape. Cylindrical cells are advantageous as there is a greater range of shorter path lengths available without the need for the cells to be demountable. Final optimisation of gelation approach inside the cell found that, after sonication of the hydrogelator suspension in a vial, the most reproducible gelation inside the cell was obtained when the thermal heating/cooling was undertaken within the cell with the use of a thermostated hot block. The cells were placed in the hot block such that the face of the cell was placed next to the hot block and any voids in the hot block were packed with aluminium foil to minimise heat loss during the process.

It is noted that the above optimisation process applied to the hydrogels of 1, $\mathbf{2}$ and $\mathbf{3}$ (A-E) and that, for different hydrogelators, a different optimisation outcome may occur i.e. one of the earlier approaches attempted may give reproducible results for a different hydrogel given the differing chemical nature of hydrogelators and corresponding self-assembly. 
3. The PMT (HV) plots for the SRCD spectra shown in Figure 5 of the manuscript.
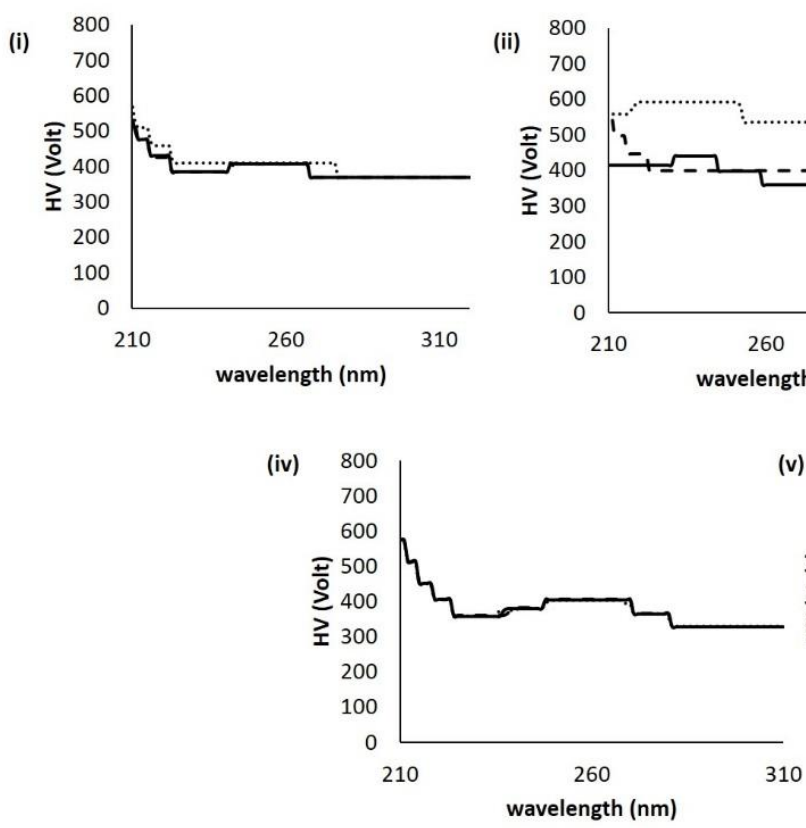

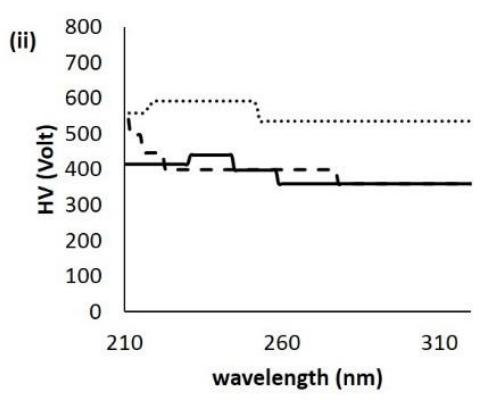

(iii)
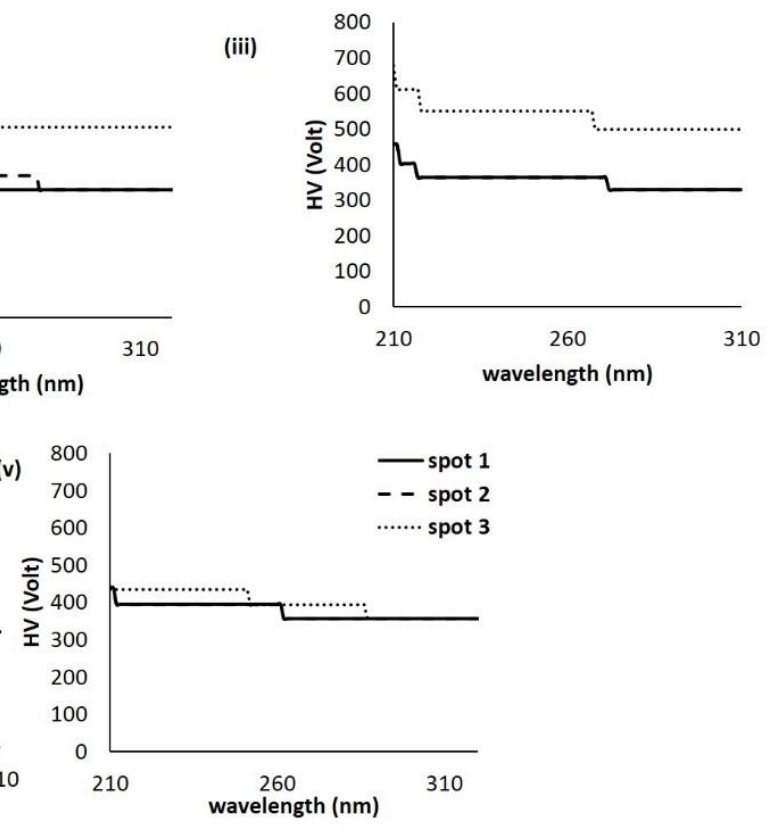

FIGURE C The PMT plots of HV for each of the SRCD spectra shown in Figure 5 i.e. obtained from different sites/spots in a hydrogel sample. (i) GalNHFmoc hydrogel A in water triggered thermally ( $0.1 \mathrm{~mm}$ PL cell); (ii) GalNHFmoc hydrogel B in PBS triggered by sonication $(0.2 \mathrm{~mm}$ PL cell); (iii) GalNHFmoc hydrogel C in PBS triggered thermally (0.1 mm PL cell); (iv) GlcNHFmoc hydrogel D in water triggered thermally (0.2 $\mathrm{mm}$ PL cell); (v) Fmoc-FF hydrogel E in PBS triggered by sonication $(0.1 \mathrm{~mm}$ PL cell). All samples were gelled in the cell of the path length (PL) stated at a concentration of $2.0 \mathrm{mg} / \mathrm{mL}$. 

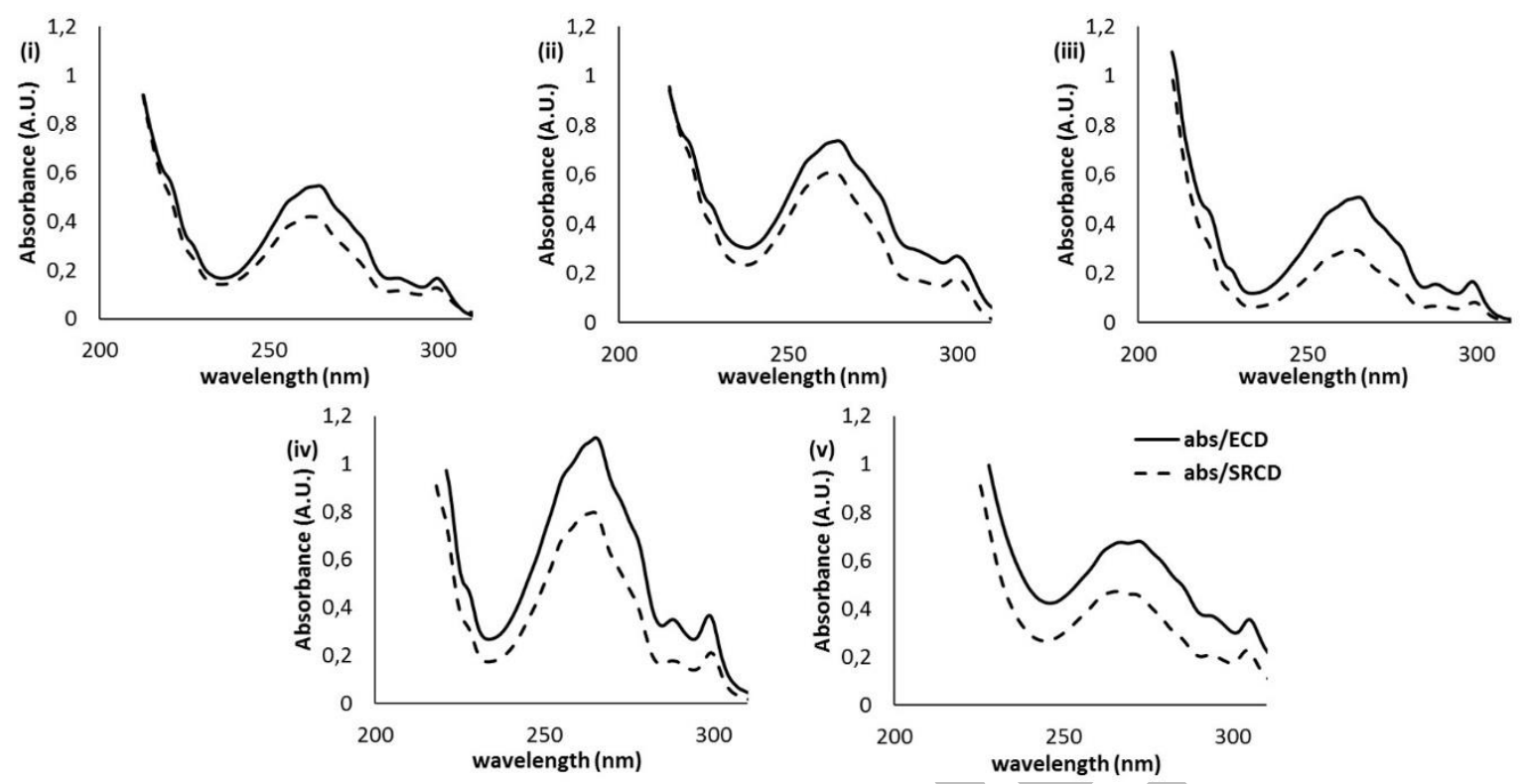

FIGURE D Comparison of the absorbance spectra from CD (Chirascan) and SRCD instruments showing the spectral differences arising from differing cross sectional areas of the beam. (i) GalNHFmoc hydrogel A in water triggered thermally (0.1 mm PL cell); (ii) GalNHFmoc hydrogel $\mathbf{B}$ in PBS triggered by sonication ( $0.2 \mathrm{~mm}$ PL cell); (iii) GalNHFmoc hydrogel $\mathbf{C}$ in PBS triggered thermally $(0.1 \mathrm{~mm}$ PL cell); (iv) GlcNHFmoc hydrogel $\mathbf{D}$ in water triggered thermally $(0.2 \mathrm{~mm}$ PL cell); (v) Fmoc-FF hydrogel $\mathbf{E}$ in PBS triggered by sonication ( $0.1 \mathrm{~mm}$ PL cell). All samples were gelled in the cell of the path length (PL) stated at a concentration of $2.0 \mathrm{mg} / \mathrm{mL}$.

\section{The absorbance spectra for the CD spectra shown in Figure 8 of the manuscript.}
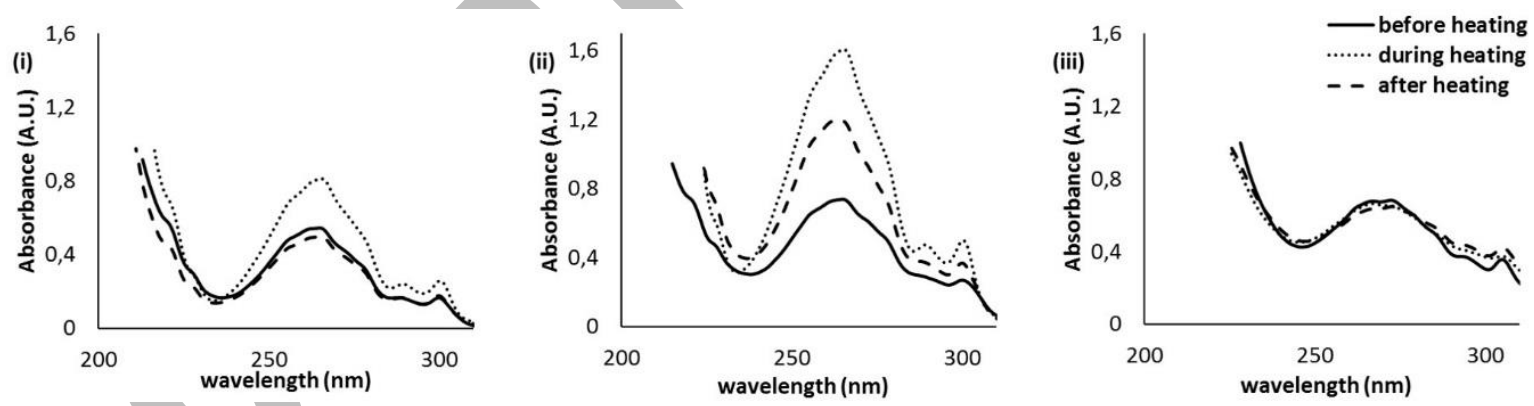

FIGURE E The absorbance spectra for the thermal studies of hydrogels to ascertain the contribution of the self-assembly to the spectrum and the reversibility of gelation. (i) GalNHFmoc hydrogel A in water triggered thermally ( $0.1 \mathrm{~mm}$ PL cell); (ii) GalNHFmoc hydrogel $\mathbf{B}$ in PBS triggered by sonication $(0.2 \mathrm{~mm}$ PL cell); (iii) Fmoc-FF hydrogel $\mathbf{E}$ in PBS triggered by sonication $(0.1 \mathrm{~mm}$ PL cell). All samples were gelled in the cell of the path length (PL) stated at a concentration of $2.0 \mathrm{mg} / \mathrm{mL}$. 